\title{
Avoiding radiation of on-demand multi-node energy charging with multiple mobile chargers
}

\author{
Tifenn Rault \\ Université de Tours, LIFAT EA 6300, CNRS, ROOT ERL CNRS 7002 \\ 64 avenue Jean Portalis, 37200 Tours.
}

\begin{abstract}
Wireless rechargeable sensor networks (WRSN) exploit wireless energy transfer techniques to replenish sensor batteries using chargers. Compared to non-rechargeable networks, WRSNs offer controllable and predictable energy replenishment and prolong the network lifetime. While previous mobile charging protocols in WRSNs consider multi-node energy transfer or the use of several chargers, in this work, we propose combining the two approaches. Our solution leverages simultaneous energy transfer to multiple nodes, with the aim of maximizing the number of nodes that are charged at each stop to reduce the charging delay. Moreover, it considers multiple chargers to serve more requesting nodes, which is expected to limit the number of node failures. Furthermore, we guarantee that there is no aggregated electromagnetic radiation (EMR), which can occur when two nearby chargers are simultaneously charging. This has not yet been considered in a mobile environment. To address these challenges, we introduce a new clustering algorithm to group demanding nodes, and we compute charging tours by iteratively solving a Traveling Salesman Problem with Multiple Time Windows problem (TSPMTW). The simulation results demonstrate that our approach reduces node failures in dynamic networks.
\end{abstract}

Index Terms-wireless rechargeable sensor networks; energy charging protocol; optimization model;

\section{INTRODUCTION}

To address the energy constraint of sensor nodes, the adoption of mobile chargers to replenish the energy supply to nodes in rechargeable sensor networks has recently garnered considerable attention in the research community [1]. Contrary to traditional energy harvesting techniques where the energy scavenged remains highly dependent on the environment since ambient energy is not always available or predictable, energy charging protocols allow for replenishing network elements in a more controllable manner.

Wireless energy transfer (WET) technologies allow pointto-point and point-to-multipoint energy transfer. In a pointto-point strategy, a charger charges only one sensor node at a time by approaching it at a very close distance so the charging process has the maximum efficiency possible. In a multi-node charging scheme, a mobile charger (MC) is able to charge multiple neighboring nodes within its charging range simultaneously, which greatly improves the charging delay and efficiency [2]. Based on which technique is used, existing charging approaches in the literature fall into three categories [3]: periodical charging, on-demand charging and collaborative charging. In periodical charging, the mobile charger periodically conducts the charging process following a preoptimized tour [4]. This supposes that the network operations are known in advance, such as the data generation rate and routing path. To address more dynamic networks when energy consumption is diverse, on-demand charging techniques aim at charging nodes with low residual energy when necessary [5]. Finally, collaborative charging schemes introduce multiple chargers to satisfy the energy demand of requesting nodes in larger networks [6].

However, wireless energy transfer introduces a new source of electromagnetic radiation (EMR) whose exposure represents a potential risk to human health [7]. Some research has been conducted to optimize the deployment of chargers with no location in the field whose EMR has exceeded a given safety threshold. In these studies, it is generally assumed that the electromagnetic radiation at a given point is proportional to the additive power received at that point [8]. Therefore, when considering multiple chargers, it is necessary to consider the aggregated electromagnetic radiation (EMR), which has not been considered yet in existing on-demand and collaborative charging schemes.

Inspired by these new findings, we propose a novel ondemand multi-node wireless charging scheme with multiple mobile chargers while guaranteeing that there is no aggregated EMR. The main contributions of our work are as follows:

- None of the existing charging schemes simultaneously consider on-demand charging, point-to-multipoint energy transfer, and multiple chargers. The present work investigates the advantage of combining these approaches in order to improve the charging performance in dynamic networks.

- We introduce a new clustering algorithm in order to fairly distribute the requesting nodes between chargers. We aim to balance both the charging load and traveled distance among chargers.

- To prevent aggregated EMR, we introduce the notion of conflicting stop locations when different chargers are in charge of nearby stop locations. Therefore, chargers must not visit conflicting stop locations at the same time.

- To limit the chargers' waiting time, we propose a path planning strategy that ensures that the visits of the chargers at conflicting stop locations are not scheduled within overlapping time windows, while minimizing the overall charging delay.

- The proposed mechanism is evaluated through simulations of on-demand scenarios. 


\section{RELATED WORKS}

In recent years, the use of multi-node charging technology in WSNs has been investigated. Xie et al. [9] considered an MC periodically traveling inside a WSN along a fixed charging path. The deployment field is partitioned into hexagonal cells, and the charger can charge sensors from the center of a cell. Their solution minimizes the energy consumption of the mobile charger by optimizing the stop duration at each cell. Then, the authors in [10] investigated the feasibility of bundling the wireless charger and the base station into a single mobile entity that simultaneously collects data and charges sensors. Their model jointly optimizes the charging schedule and flow routing. By the end, the charging tour, flow routing, and energy consumption at each node are all cyclic. In [11], the authors identified the optimal velocity control of a mobile charger as a key design objective and proposed a nearly optimal solution. The study in [12] proposed two charging algorithms which aim at shortening charging time and distance via merging and splitting charging missions. However, these solutions are based on offline scheduling, and the charging task is performed periodically. Thus, information such as timely energy status is assumed to be known in advance by MCs.

Non-deterministic charging schemes attempt to propose practical solutions to highly dynamic and unpredictable networks. In an on-demand charging scheme, when the residual energy of a node falls below a certain threshold, it will initiate a charging request to the MC. Reference [13] proposed an online solution that groups the requesting sensors into different clusters according to their locations. The charger then evaluates each cluster with a metric called the charging gain and serves the cluster with the highest charging gain. In [14], the authors presented three new protocols assuming different levels of network knowledge and introduced "criticality" to capture a node's importance in the network, which relies on the traffic served by the node and the energy consumed by the node. The study in [15] aims to reduce both the travel distance and charging delay by clustering sensors according to their energy consumption rates. Then, a collection of TSP tours is constructed for each cluster, and the charger selects the shortest TSP tour involving devices with low residual energy. Recent references investigated the possibility to partially charge the sensors in on-demand architecture [16]-[18]. This enables more sensors to be charged by the MC before its energy depletes, but it leads to more frequent charging requests and increases the traveling distance. Khelladi et al. [5] introduced OMC, a charging strategy that takes advantage of simultaneous multi-node energy transfer. OMC groups charging requests and plans a minimum-stop path for the charging tour. The authors showed that minimizing the number of stops maximizes the number of nodes that are simultaneously charged, thus reducing node failures. These studies consider either point-topoint or point-to-multipoint energy transfer, but only with one charger.

In an attempt to further improve charging solutions, some research efforts have proposed the use of multiple chargers.
Compared with the single-charger approach, using several MCs is expected to increase the scalability of the solutions but also involves coordination among mobile chargers. Authors in [19] studied how to minimize the total traveling cost of multiple chargers while ensuring that there are no node failure occurences and deriving theoretical results on the minimum number of mobile vehicles required for perpetual network operations. Then, they devised a heuristic algorithm, which selects the nodes to recharge according to the weighted sum of the travel time and residual lifetime of the sensor nodes. Reference [20] considered the multiple chargers scheduling problem for event-driven applications. The solution objective is to maximize the coverage since every sensor monitors a circular area. Madhja et al. [6] proposed centralized and distributed protocols for efficient charging assuming different levels of network knowledge (from global to local and with no network knowledge). During the coordination phase, each $\mathrm{MC}$ is assigned to a network region, and during the charging phase, each charger traverses the region to which it is assigned to charge the sensors. In their model the charging is performed point-to-point, and the charging time is assumed to be equal for every sensor and independent of its battery status. Lin et al. [3] considered a charging scheme where multiple mobile chargers can charge either a sensor or another mobile charger. They proposed a game theory-based solution that seeks to maximize the charging efficiency of the network. At the beginning of each round, each $\mathrm{MC}$ is assigned a service region containing sensors and chargers needing to be charged. It then computes the charging priorities based on the sensor's (or charger's) residual energy and their contribution to the network. In [21], the authors jointly optimized depot positioning and charging tour planning for multiple MCs. They maximized the ratio of time spent on charging over time spent on traveling, while reducing the number of required charging tours, MCs and charging depots.

However, when considering multiple chargers, it is necessary consider electromagnetic radiation (EMR) safety issues. It has been shown that exposure to high levels of electromagnetic radiation can present a threat to human health. Given a set of stationary chargers, Dai et al. studied in [8] how to select which chargers to turn on so that nowhere on the planar field exposes electromagnetic radiation exceeding a certain limit. The authors extended their work in [22] by considering the transmitting power of the chargers to be adjustable instead of an on/off operation. In [23], the authors proposed a wireless charger placement scheme that guarantees EMR safety for every location on the plane. The study in [7] optimizes the amount of "useful" energy transferred from stationary chargers to nodes (under constraints on the maximum level of radiation). Thus, existing approaches optimize the placement or the scheduling energy transfer of static chargers, not mobile chargers. However, in an on-demand charging scenario with multiple chargers, there is aggregated EMR. That is, nearby chargers transfer energy simultaneously to their own subset of nodes, and there are locations receiving aggregated EMR.

Table I summarizes the features considered by reviewed 
charging protocols. Our approach departs from the existing on-demand charging solution since we consider simultaneous energy transfer, multiple mobile chargers, and aggregated electromagnetic radiation.

TABLE I

REVIEWED CHARGING SCHEMES.

\begin{tabular}{|c|c|c|c|c|}
\hline Ref & $\begin{array}{c}\text { on-demand } \\
\text { charging } \\
\text { scheme }\end{array}$ & $\begin{array}{c}\text { multi-node } \\
\text { charging }\end{array}$ & $\begin{array}{c}\text { multiple } \\
\text { mobile } \\
\text { chargers }\end{array}$ & $\begin{array}{c}\text { radiation } \\
\text { constrained } \\
\text { scheme }\end{array}$ \\
\hline$[9]-[12]$ & no & yes & no & no \\
{$[13]-[18]$} & yes & no & no & no \\
{$[5]$} & yes & yes & no & no \\
{$[3],[6],[19],[20]$} & yes & no & yes & no \\
{$[21]$} & no & no & yes & no \\
{$[7],[8],[22],[23]$} & no & yes & no & yes \\
\hline
\end{tabular}

\section{OUR SYSTEM MODEL}

\section{A. Network model}

We consider a network with $K$ mobile chargers (MCs) and a set of static sensor nodes that are randomly deployed within a two-dimensional area. Sensors are powered by an on-board battery of limited capacity. When their energy level falls below a certain threshold, nodes send out requests to one of the mobile chargers. A charger then stores the received charging requests and decides when to launch a new charging tour. Based on the collected energy requests, it then computes the charging route of every charger and informs them.

\section{B. Energy model}

Nodes consume energy for sensing, communications and computational tasks, but we assume that the energy consumption profile is not known in advance. Depending on the underlying application or routing protocol, nodes may exhibit a diverse energy status. A charger can perform its charging function when it stops somewhere in the deployment field. Each charger has a power charging disk of radius $R_{c}$, as illustrated in Figure 1. It can simultaneously recharge multiple sensors if they are located within its power charging disk with the following charging model [5], [9], [10]:

Let $h$ be one of the stop locations in the charging path of charger $k$. The received power $p_{i}(h)$ by a sensor $i$ located at a distance $d_{i, h}$ from the stop location is:

$$
p_{i}(h)=\mu\left(d_{i, h}\right) p_{t},
$$

where $p_{t}$ denotes the charger's transmission power and $\mu\left(d_{i, h}\right)$ is the efficiency of the wireless charging process. Note that $\mu\left(d_{i, h}\right)=0$ if $d_{i, h} \geq R_{c}$. To fully charge a node battery of capacity $C_{i}$, the charging time $t_{i}(h)$ is:

$$
t_{i}(h)=\frac{C_{i}}{p_{i}(h)}
$$

Finally, the stop duration time $e_{h}$ of the charger at stop location $h$ is the time required to recharge all the sensors covered by the stop location, and can be expressed by:

$$
\text { time }_{h}=\max _{i \mid d(i, h) \leq R_{c}}\left(t_{i}(h)\right)
$$

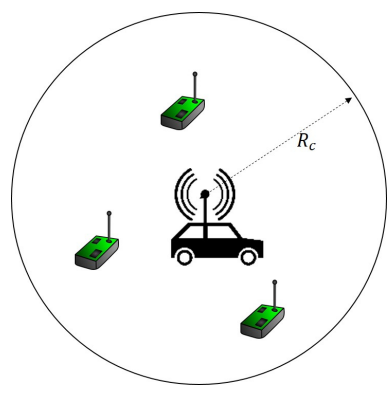

Fig. 1. Power charging disk

\section{Aggregated EMR model}

The aggregated EMR from multiple chargers is generally assumed to be the sum of each charger's EMR, and the intensity of the EMR at a location is proportional to the received power there [8], [23]. To ensure EMR safety, existing processes guarantee that at any location, the aggregated EMR does not exceed a given threshold. For simplicity, we want to prevent any aggregated EMR from occurring, which is also more conservative. Given our energy model, there is aggregated EMR if the distance between the two charging chargers is less than $2 \times R_{c}$, i.e., if their power charging disks intersect.

\section{ON-DEMAND CHARGING PROTOCOL}

Our charging solution includes three steps, as summarized in Figure 2. First, our solution collects charging requests in order to group them in the upcoming charging tour. To decide when to start a new charging tour and which stop locations must be visited, we use the mechanisms proposed in [5]. Then, we cluster the stop locations using our clustering algorithm in order to balance the charging loads between the chargers. Finally, we compute a charging route for each mobile charger, guaranteeing that there is no aggregated EMR while minimizing the tour duration. Note that we make a distinction between a charging tour and a charging route: a charging tour includes $K$ charging routes, one for each charger.

A. Step 1 - Threshold-based tour launching, stop locations, and determination of durations

Here, we use the mechanim proposed by Khelladi et al. in [5]. The purpose of their work is to take advantage of simultaneous energy transfer in two ways.

- First, the threshold-based tour launching mechanism waits for a maximum number of charging requests in order to serve a higher number of nodes in each charging tour. In short, the solution uses two thresholds, $L_{c}$ and $L_{r}$, with $L_{c} \leq L_{r}$. Nodes send a request message to the MC if their energy level falls below $L_{r}$. Nodes also send an 


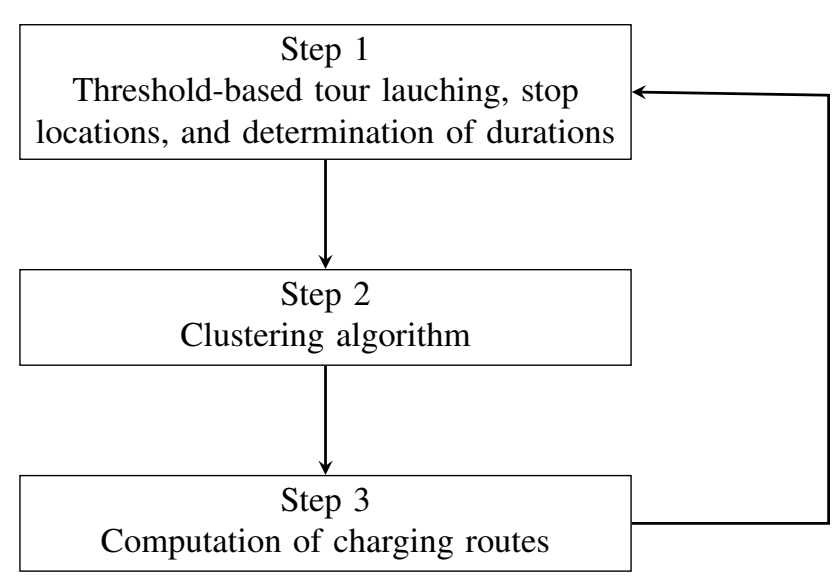

Fig. 2. Protocol overview.

alert message if their energy level falls below $L_{c}$. Upon receiving the first alert message, the MC starts a new charging tour including all the nodes that have issued a request message. For more details, the reader is referred to [5].

- Second, they determine the minimum number of stop locations the charger will have to visit. Indeed, minimizing the number of stops is expected to maximize the number of nodes that are charged simultaneously. The authors solve this problem using a clique partition based problem.

- Third, the stop duration at a stop location corresponds to the time required to fully charge the requesting nodes covered by the stop location. This duration depends on the nodes' battery levels and on the distances separating the nodes from the stop location.

\section{B. Step 2 - Clustering algorithm}

When a tour is required, we need to cluster the stop locations into $K$ groups so that each charger can recharge its own subset of nodes. Our objective is to balance the charging load between the chargers while minimizing the traveled distance. This is because we assume the chargers have identical charging capacity, so we apply a fair distribution of charging tasks. Moreover, it is expected that the chargers will end their charging route nearly simultaneously, thus reducing the charging delay.

Our algorithm is summarized in Algorithm 1. We first randomly select a cluster head for each charger. Then, we iteratively add the closest demanding node to the cluster with the minimum accumulated charging time. The rationale behind this algorithm is to group stops that are geographically close in order to reduce the traveled distance inside a cluster while guaranteeing that the charging times are equal between chargers.

\section{Step 3 - Computation of charging routes}

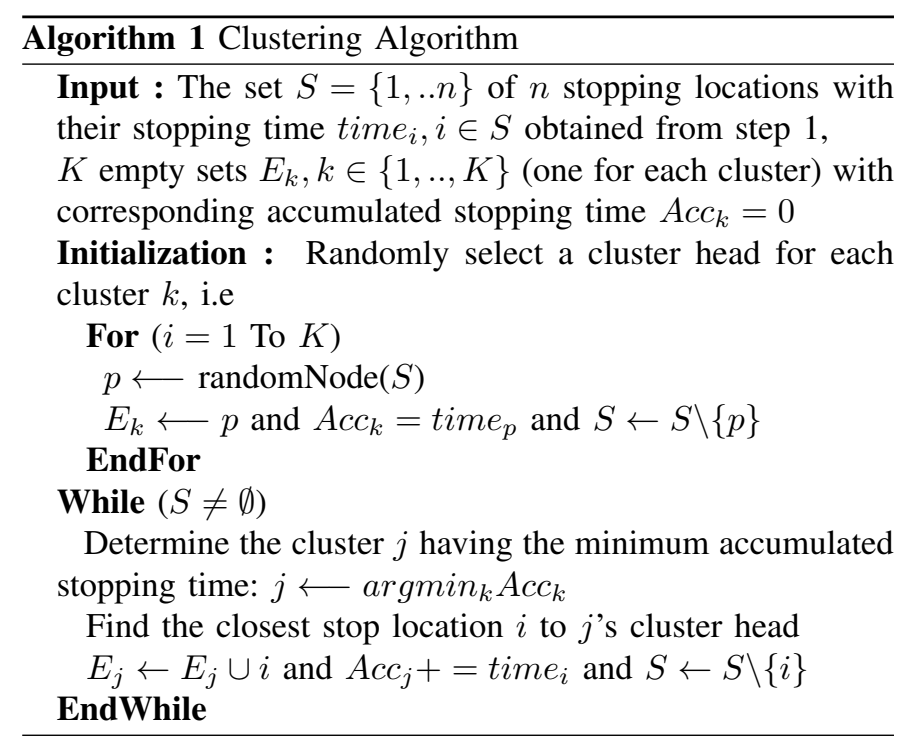

Our objective is to compute a charging route for each MC (visiting order of the stop locations and stop duration) while ensuring that there is no aggregated EMR. To do this, we introduce the notion of conflicting stop locations: two stop locations $i$ and $j$ are conflicting if $d(i, j) \leq 2 * R_{c}$, and $i$ and $j$ are affected by different MCs. Thus, if a charger visits $i$ while another simultaneously visits $j$, there will be aggregated EMR, since the two nearby chargers will transmit power at the same time.

Therefore, we want to compute $K$ routes while ensuring that two conflicting stop locations are not visited at the same time by different chargers. To tackle this problem, we were interested in a particular class of TSP problems called the Traveling Salesman Problem with Multiple Time Windows (TSPMTW). In the TSPMTW, every customer can specify a number of time windows in which the delivery can start [24], [25]. We first provide insights about the TSPMTW problem. We then explain how it is used in our solution to compute the charging routes.

\section{1) Definition of the TSPMTW problem:}

Let $I=1, \ldots, n$ be the set of stop locations $i \in I$ that must be visited by a charger. Every stop location $i \in N$ has a set of $w_{i}$ time windows $\left[e_{i}^{v}, l_{i}^{v}\right], 1 \leq v \leq w_{i}$, such that $e_{i}^{v}<l_{i}^{v}$ and $l_{i}^{v}<e_{i}^{v+1}$. The service of stop $i$ should start in one of these time windows. Moreover, each location $i$ has a stopping time time $_{i}$ corresponding to the time needed to fully charge the nodes covered by the stop location. Let 0 be a depot at which the route must start and end. For each arc $(i, j) \in I \cup 0$, we have a time $t_{i j}$. For $i \in I, t_{i j}$ includes the stop duration time $_{i}$ at stop $i$ and the travel time to go from $i$ to $j$, while $t_{0 j}$ only includes the travel time from the depot to stop $j$. The minimum route duration of TSPMTW problem is an issue of finding the optimal sequence of stop locations such that

(1) the time of arrival of the charger at the depot is minimized;

(2) every stop location is served once by the charger; 
(3) the service of every stop location starts within one of its time windows; if the charger arrives before the beginning of the time window, it must then wait;

Note that the objective function of the TSPMTW problem can be to minimize the traveled distance cost [26], the route duration [24], or a combination of both [25]. In our case, we are interested in minimizing the tour duration in order to minimize the charging delay. Indeed, a naive solution to prevent aggregated EMR consists of making chargers wait when they arrive at a conflicting stop location if another charger is already charging. When using this mechanism, we can compute a TSP-based solution and wait if necessary when visiting the stop locations. However, this approach incurs a waiting time during which the chargers are neither traveling nor charging, which will increase the charging delay and node failures. To limit the chargers' waiting time, a minimum route duration TSPMTW-based path planning strategy ensures that the visits of the chargers at conflicting stop locations are not scheduled within overlapping time windows, while minimizing the overall charging delay.

In literature, the TSPMTW problem is solved using either linear programming-based heuristics [24], [25] or constraint programming models [26]. In this paper, we used the vehicle routing library provided by the OR-Tools suite, which relies on a constraint solver [27].

\section{2) Our solution relies on the following TSPMTW problem:}

Thanks to this approach, we can add time windows to the stop locations in order to specify when these stop locations can be visited. Typically, we will remove time windows at conflicting stops to capture the fact that they cannot be visited during given periods. Our idea is to compute each charging route one by one, and when we consider the $k^{\text {th }}$ charging route we modify the time windows of stops that conflict with already scheduled stop locations.

The general procedure is given in Algorithm 2. The route of the first charger is computed using a TSP: at that point the first charger does not conflict with another charger, so there is no time window on the stop locations of the first charger. Then, when the $k^{t h}(k \in[2, K])$ charger computes its route, it first computes the time windows of its stop locations, following Algorithm 3. To do that, the $k^{t h}$ charger knows the charging routes of the $k-1$ chargers since they have already computed their route. For all its stop locations $i$, charger $k$ checks if there are scheduled locations $j$ conflicting with $i$. If so, charger $k$ knows that its stop $i$ cannot be visited during the service duration of $j$. Therefore, the time windows of $i$ are updated accordingly. It is important to note how we compute the length of the time of windows that are removed. For example, if location $j$ is visited by charger $c$ at time $d_{j}, c$ will stay at $j$ for time $_{j}$ units of time. Then, if a location $i$ must be visited by charger $k$, and if $i$ conflicts with location $j$, the charger $k$ cannot start its service within $\left[d_{j}-t_{i m e}, d_{j}+t_{i m e}\right]$. Finally, given its stop locations and their time windows, the $k^{\text {th }}$ charger determines its route solving a TSPMTW problem.
For better understanding, the behavior of Algorithm 2 is illustrated in Figure 3. We assume in this example that the travel time between two nodes is equal to 5 units of time, and the service time at each node is equal to 10 .
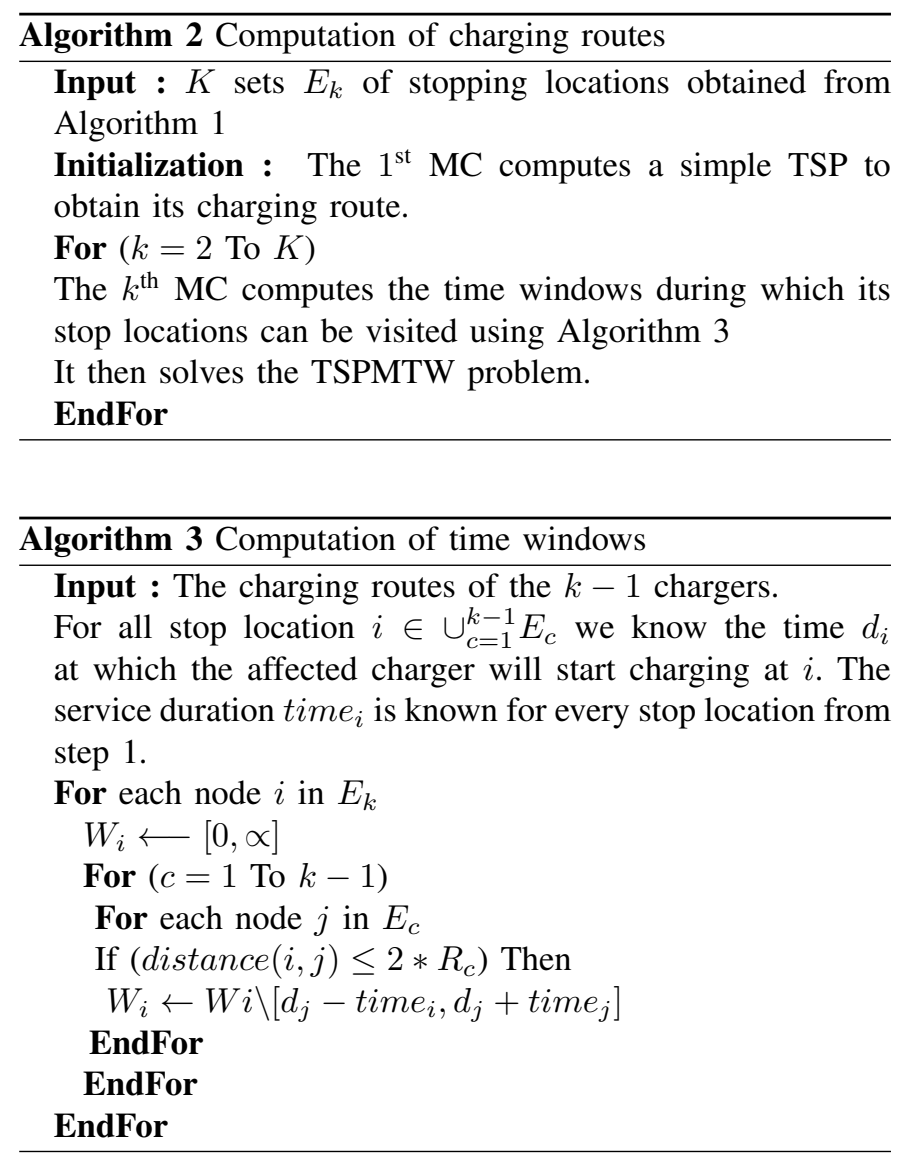

\section{Computation time complexity}

Here, we provide a computation time analysis for the worstcase scenario of our solution. Let's detail the time complexity of each step.

Time complexity of step 1: The time complexity of this step is $\mathcal{O}\left(n^{3}\right)$ according to [5].

Time complexity of step 2: The time complexity of our clustering algorithm is $\mathcal{O}\left(n^{2}\right)$. Indeed, in Algorithm 1, the initialization loop runs in $\mathcal{O}(K)$ time in order to randomly select a cluster head for each cluster. The main loop runs in $\mathcal{O}(n \cdot(K+n))$ time. If we assume $K \ll n$, the global complexity of step 2 is $\mathcal{O}\left(n^{2}\right)$.

Time complexity of step 3 (cf Algorithm 2): We denote $n_{k}$ as the number of stop locations in cluster $k$.

Initialization phase: This phase consists in solving a TSP on cluster 1, which is strongly NP-hard. In this paper, the problem is solved exactly with an exponential algorithm since $n_{1}$ is small, but it could be solved in polynomial time using a heuristic (e.g. $\mathcal{O}\left(n_{1}^{2}\right)$ using a nearest neighbor heuristic).

Updating time windows: Updating the time windows at iteration $k$ costs $\mathcal{O}\left(n_{k} \cdot\left(\sum_{c=1}^{k-1} n_{c}\right)\right)$. Since this operation 


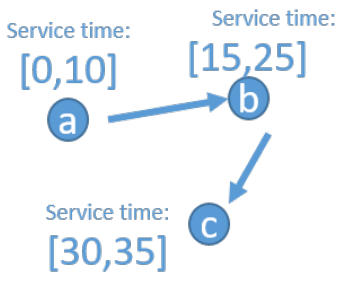

(a) The $1^{\text {st }} \mathrm{MC}$ computes its charging tour using a TSP. The resulting service times are displayed.

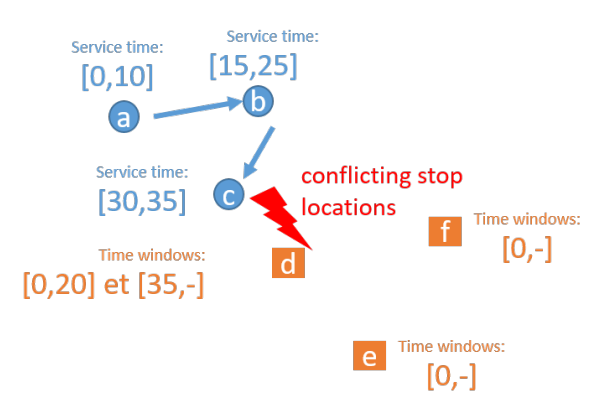

(b) The $2^{\text {nd }} \mathrm{MC}$ identifies the conflicting nodes between its nodes and those of the $1^{\text {st }}$ MC. The $2^{\text {nd }}$ MC then computes the available time windows of its nodes. We can see that node $d$ cannot be visited when $c$ is visited by the $1^{\text {st }}$ charger since $c$ and $d$ are conflicting nodes.

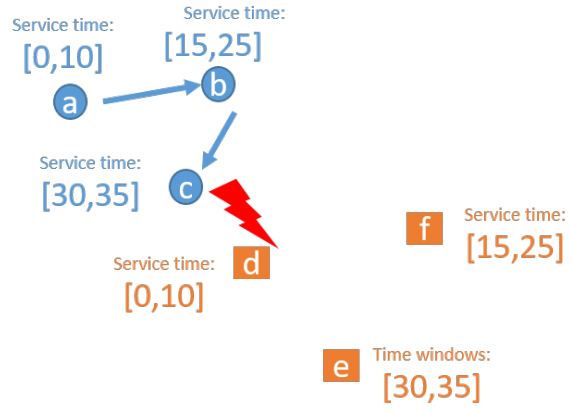

(c) The $2^{\text {nd }}$ MC solves the TSPMTW to obtain a charging tour that respects the constraints on the time windows. Here, we can see that node $d$ is not visited during the service time of $c$.

Fig. 3. Illustration of how the charging routes are computed.

is performed $K-1$ times, it will cost $\mathcal{O}\left(\sum_{k=2}^{K} n_{k}\right.$. $\left.\left(\sum_{c=1}^{k-1} n_{c}\right)\right)$. Since $n_{k}$ and $n_{c}$ are bounded by $n$, this phase runs in $\mathcal{O}\left(K^{2} n^{2}\right)$ time. This is a polynomial time complexity because we have $K \ll n$.

Solving TSPMTW problems: In this paper, we used an exact method with an exponential time complexity to solve the TSPMTW problems. However, we show in what follows that we can achieve a polynomial time complexity using a heuristic. Indeed, the authors in [25] proposed a heuristic solution with an overall $\mathcal{O}\left(\mathrm{m}^{3} w\right)$ time complexity, where $m$ is the number of visited stops, and $w$ is the total number of time windows. Using their method to run the $K-1$ TSPMTW will cost $\mathcal{O}\left(\sum_{k=2}^{K} n_{k}^{3} \cdot w_{k}\right)$, where $w_{k}$ is the total number of time windows in cluster $k . w_{k}$ is bounded by $\mathcal{O}\left(n_{k} \sum_{j=1}^{k-1} n_{j}\right)$. The bound is reached when all the stop locations in cluster $k$ are conflicting with all the stop locations in clusters 1 to $k-1$. Therefore, the time complexity can be rewritten $\mathcal{O}\left(\sum_{k=2}^{K} n_{k}^{3} \cdot\left(n_{k} \sum_{j=1}^{k-1} n_{j}\right)\right)=\mathcal{O}\left(\sum_{k=2}^{K} n_{k}^{4}\right.$. $\left.\left(n_{k} \sum_{j=1}^{k-1} n_{j}\right)\right)$. Since all $n_{k}$ are bounded by $\mathcal{O}(n)$, this phase runs in $\mathcal{O}\left(K^{2} n^{5}\right)$ time. Note that since $K \ll n$, this phase has a polynomial time complexity.

Overall time complexity: The computation time of our solution is dominated by the computation of the charging routes (step 3). As a result, the global time complexity is $\mathcal{O}\left(K^{2} n^{5}\right)$.

\section{Performance evaluation}

The performance evaluation is conducted in two stages. First the proposed strategy is simulated in offline scenarios, where each experiment has a fixed number of requesting nodes for which a charging tour is planned. In the second stage, the entire charging protocol is evaluated in real-time, on-demand scenarios.

We compare our charging protocol against the three following protocols:
- OMC [5]. In OMC, there is only one charger, so there is no aggregated EMR. However, it is expected that our solution scales better because we consider multiple mobile chargers.

- CCGK [6]. The CCGK (Centralized Coordination Global Knowledge) protocol considers multiple chargers geographically distributed in the network. During the coordination phase, each sensor chooses the closest charger with the highest remaining energy. During the charging phase, a charger will first charge nodes with a low energy and short distance. CCGK does not consider multi-node energy transfer, but for fairness, we apply this approach once the stop locations are computed after step 1. As a result, CCGK can be seen as an alternative strategy for the clustering phase and the charging tour computation phase.

- TSP. Since there is no study that simultaneously consider multiple chargers, on-demand charging, multi-node energy transfer and radiation constraints, we also compare our solution against a modified version of our charging protocol. Instead of solving TSPMTW problems to determine the charging paths, we propose solving TSP problems to study the impact of the charging tour on the system performance. The TSP-based solution does not inherently consider the EMR safety issue since it only seeks to minimize the distance traveled by chargers. Therefore, in the TSP-based solution, chargers will have to wait when they arrive at a conflicting stop location if another charger is already charging in order to guarantee the absence of aggregated EMR. It is expected that using TSP will lead to reduced traveled distances but higher waiting times at conflicting locations, thus resulting in an increased charging delay and node failures.

\section{A. Simulation settings}

For each simulation, sensors are randomly deployed within a $25 \times 25 \mathrm{~m}^{2}$ square area. They each have equal battery capacity 
$C_{i}=50 \mathrm{~J}$ and different initial energy levels evenly distributed between 0 and $C_{i}$. The charging efficiency function $\mu(d)$ was computed by [5], [9], [10] using the experimental measurements on the efficiency of simultaneous energy transfer provided by Kurs et al. in [2]. Through curve fitting the experiment results, they obtained $\mu(d)=-0.0958 d^{2}-0.0377 d+1.0$. Assuming the chargers have a transmission power $p_{t}=5 \mathrm{~W}$, and a minimum received power $(1 W)$ that allows a sensor to be charged, the maximum charging range is $r_{c}=2.7 \mathrm{~m}$. The mobile chargers speed is $v=2 \mathrm{~m} / \mathrm{s}^{2}$, and their traveling power consumption $p_{\text {travel }}=7.4 v+0.29$, as in [5].

\section{B. Offline simulation results}

In the offline scenario, we consider a fixed number of requesting nodes and the corresponding charging routes are computed. The objective is to evaluate the effectiveness of our approach neglecting the dynamics of charging requests arrivals.

We first study the average number of stop locations per charger, as calculated by steps 1 and 2 of our charging protocol. As shown in Figure 4, the number of stop locations per charger increases with the number of requesting nodes, and the more chargers there are available, the lower the number of stops. This is because stop locations are dispatched between a larger number of chargers. Thanks to this figure, we can see that with 300 requesting nodes, the number of stop locations is equal to 52 when there are two chargers. Therefore, in this case, compared to a point-to-point energy transfer strategy, our solution reduces the number of stops by one-third. As a consequence, it makes the TSP/TSPMTW problems more tractable since there are fewer customers to visit. The gain is even greater when considering more nodes: with 1000 requesting nodes, the number of stop locations is equal to 160 for two chargers, reducing the number of stops by 6 . When the density increases, there are more opportunities to group sensors.

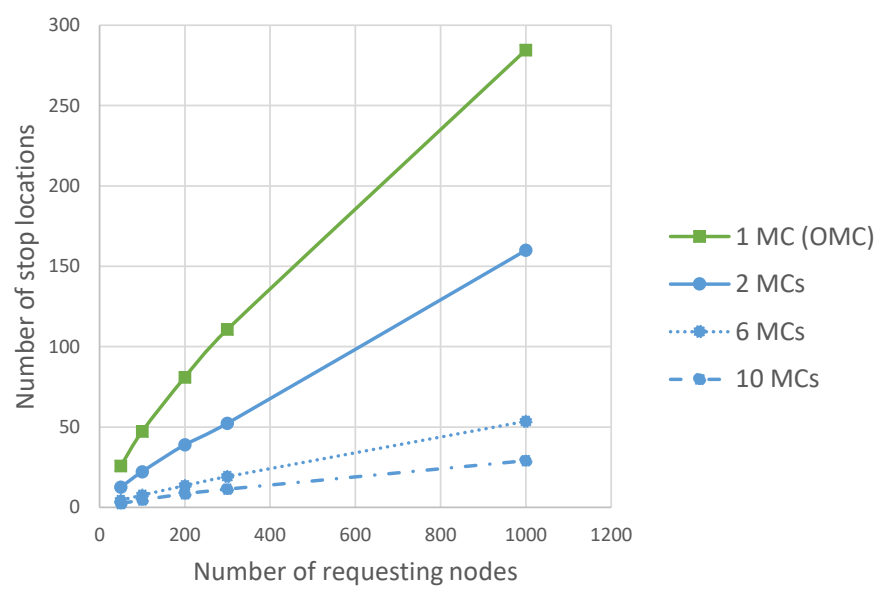

Fig. 4. Number of stop locations per chargers.

To better understand further results, we study the characteristics of the clusters obtained by our clustering algorithm against the ones obtained by CCGK. In Figure 5, we plot the standard deviation of the charging time between clusters. As seen, CCGK exhibits the highest charging time disparity between clusters. This is because our approach prioritizes the charging load distribution, while CCGK tends to build spatially distributed groups. Therefore, CCGK can create clusters with very different charging times. As illustrated in Figure 6, with CCGK, a charger can have a charging time up to 5 times longer than another charger. In contrast, our clustering algorithm creates clusters with equal charging times.

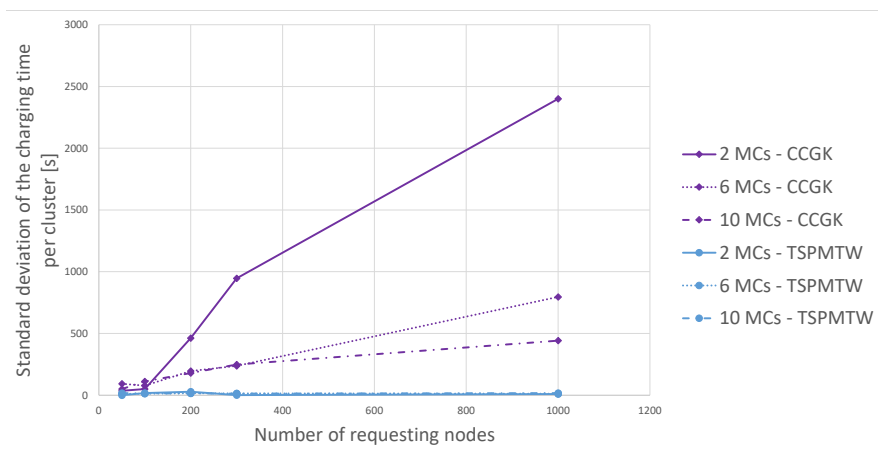

Fig. 5. Standard deviation of the charging time per charger.

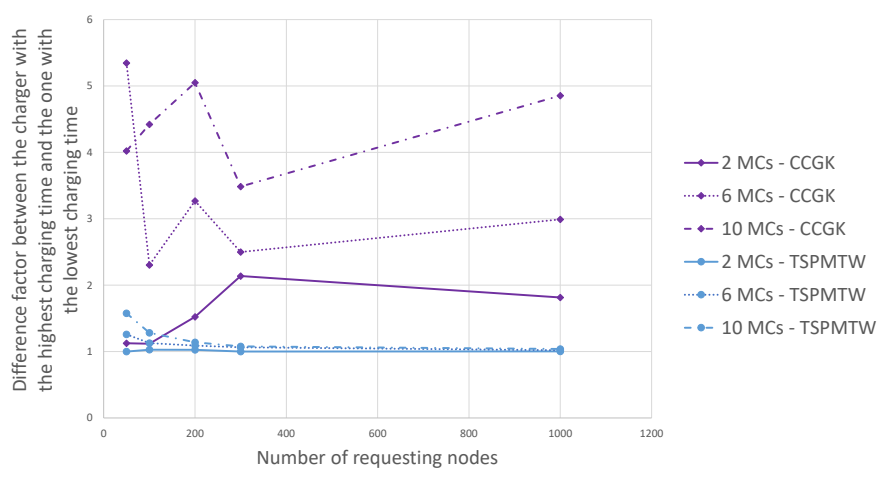

Fig. 6. Factor difference between chargers' charging time.

We then measure the distance from the stop locations to the "center of gravity" of their cluster. As expected, we can observe in Figure 7 that with our clustering algorithm, the visited locations inside a cluster are more distant from each other. As a consequence, we will see later that CCGK tends to produce charging routes with shorter traveled distances but higher charging durations.

Only the CCGK protocol leads to aggregated EMR. Indeed, in OMC, there is only one charger, and in the TSP and TSPMTW solutions, chargers wait if they arrive at a conflicting stop location and if another charger is already charging. We show in Figure 8 the percentage of the time of the tour during which there is aggregated EMR. It increases as the number of chargers increases since there are more chargers sharing the spatial area. Moreover, we can see that for 10 chargers, there is aggregated EMR almost $50 \%$ of the time. This shows that in a mobile environment with multiple mobile chargers, there 


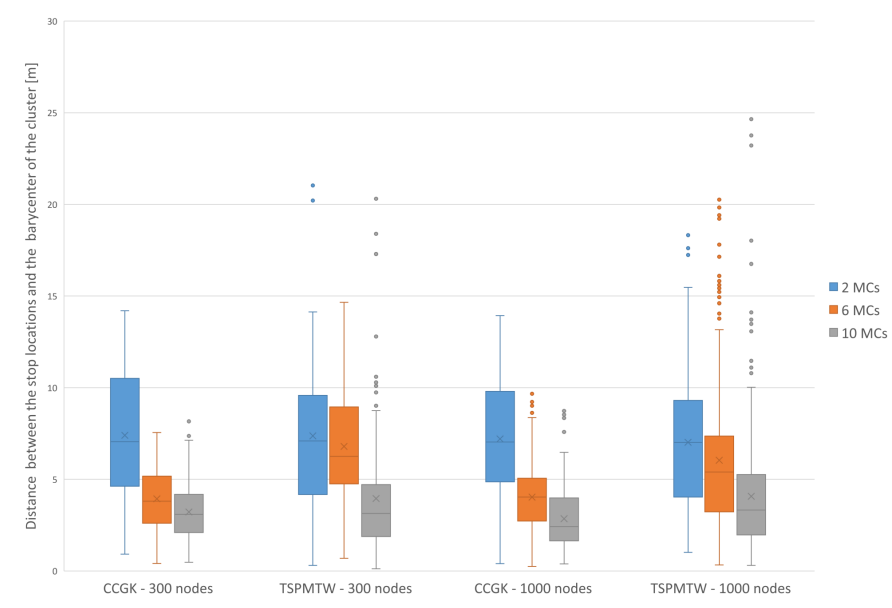

Fig. 7. Spatial distribution of the stop locations inside clusters.

is aggregated EMR, which has to be taken into account when designing charging protocols.

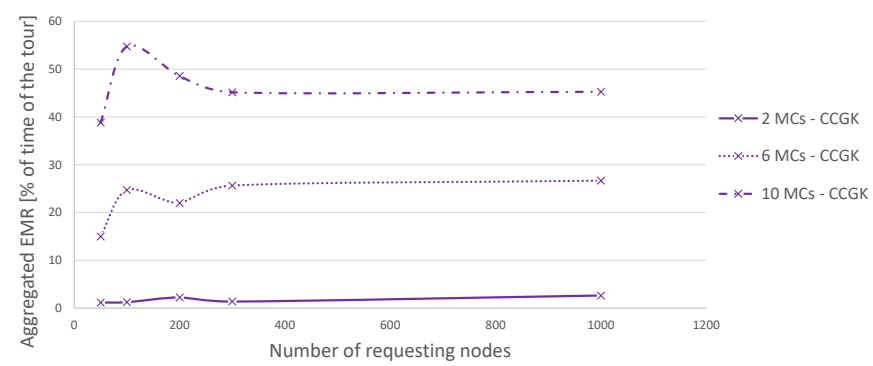

Fig. 8. Aggregated EMR during a charging tour.

In Figure 9, we plot the charging tour duration of the different approaches as a function of various numbers of MCs and network sizes. The charging tour duration is the duration of the longest charging route time. It can be seen that when the number of requesting nodes increases, the charging tour becomes longer. It is directly related with Figure 4 since there are more stop locations to visit. Inversely, the introduction of additional chargers allows reducing the charging tour duration. CCGK exhibits a performance between the TSP and TSPMTW solutions. On the one hand, TSP/TSPMTW protocols introduce a waiting time to prevent aggregated EMR, but chargers have nearly equal charging times. On the other hand, CCGK does not introduce a waiting time but builds clusters with uneven charging times. As a result, the absence of a waiting time is compensated by routes with longer charging duration. Finally, compared to other approaches, the TSPMTW-based charging protocol minimizes the end time of the charging tour.

This improvement in terms of tour duration comes at the cost of an increased traveled distance. To reduce the waiting time, a charger may not follow the shortest path. As seen in Figure 10, with a TSPMTW-based protocol, chargers travel longer distances compared to the TSP-based solution. We believe it is acceptable to slightly increase the energy consumed

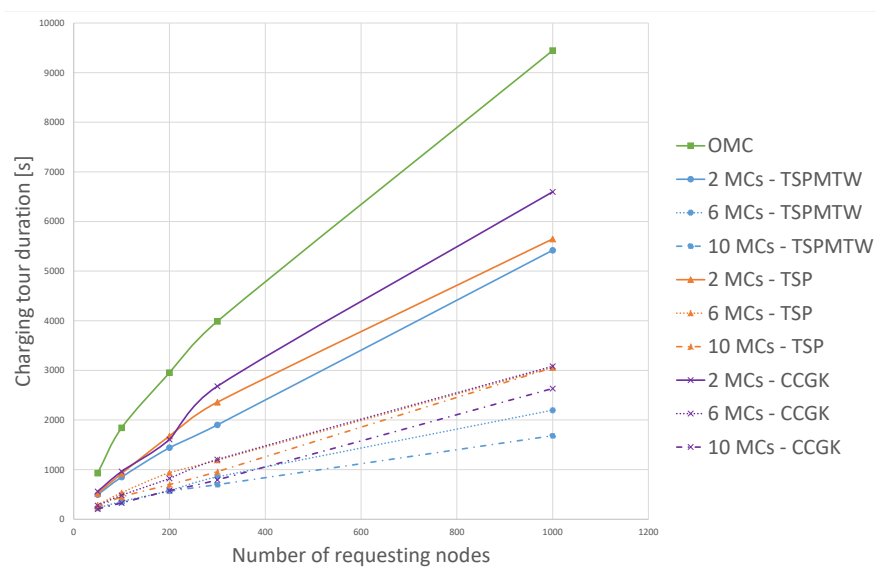

Fig. 9. Charging tour duration.

by the chargers for traveling in order to reduce the charging delay. We plot in Figure 11 (resp. Figure 12) the energy spent by chargers for traveling (resp. charging). As seen, the energy consumed for traveling does not represent the biggest part of the energy spent by chargers. In our experiments, the energy used for charging is 3 to 13 times higher than the energy for traveling.

Regarding CCGK, it has a short traveled distance for 6 and 10 chargers, while it has the longest traveled distance for 2 chargers. This can be explained by the fact that at the beginning, CCGK creates well geographically distributed clusters. Therefore, compared to our clustering algorithm, stop locations inside a cluster are closer, and one can imagine that routes will be shorter. However, this gain in space clustering is mitigated by the way chargers build their tour: a charger moves to the node that minimizes the product of the node's energy times its distance from the current position of the charger. Therefore, when there are only two chargers, the way the tour is built strongly penalizes the tour distance, as there must be some back-and-forth movements. Finally, the OMC solution exhibits the highest traveled distance: it is explained by the fact that in the OMC solution there is only one charger that has to assume the whole charging task.

Figure 13 shows the time required to compute the minimum number of stop locations and to cluster these stops. We can see that the computation time of steps 1 and 2 of our protocol mainly depends on the number of nodes that need to be charged. Indeed, the computation time complexity of step 1 is $\mathcal{O}\left(n^{3}\right)$ according to [5], while our clustering algorithm runs in $\mathcal{O}\left(n^{2}\right)$. Therefore, the time complexities of phase 1 and 2 are dominated by the number of requesting nodes.

The computation time required to solve the $K$ TSPMTW problems at step 3 (see Figure 14) is influenced by both the number of mobile chargers and the number of requesting nodes, as studied in subsection IV-D. As the number of charged nodes increases, the charging paths include more stop locations, thus increasing the computation time of one TSPMTW problem. However, as the number of chargers increases, we compute a higher number of TSPMTWs (one for 


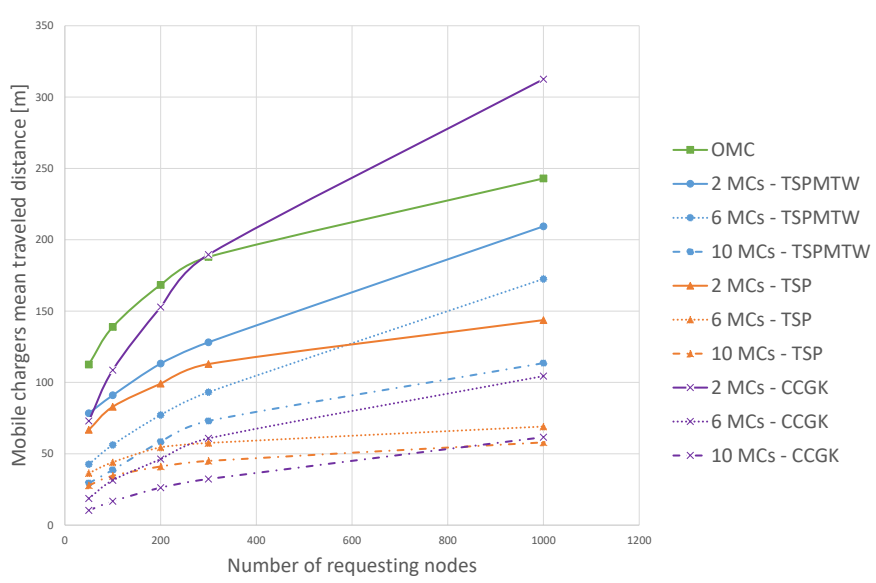

Fig. 10. Distance traveled by chargers during a charging tour.

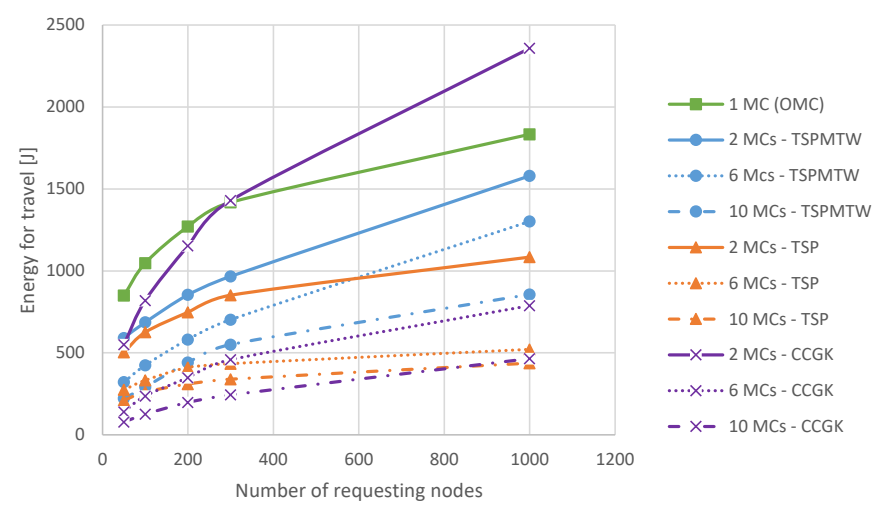

Fig. 11. Energy spent by chargers for traveling.

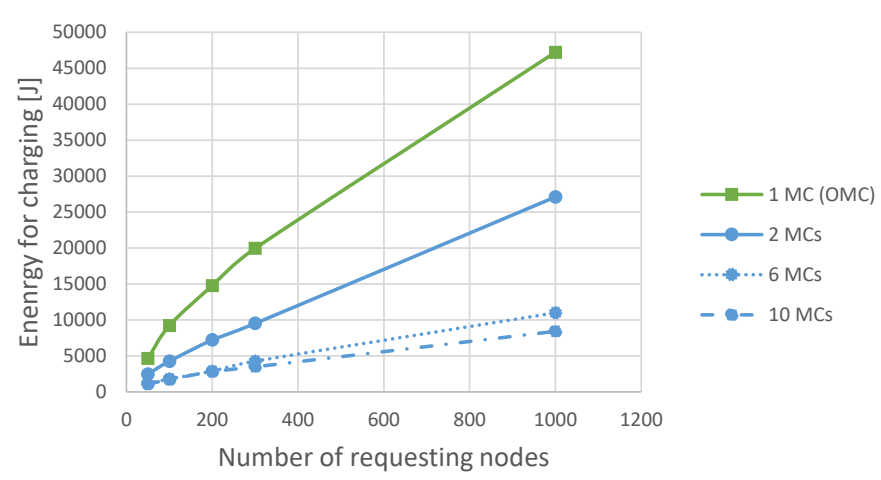

Fig. 12. Energy spent by chargers for charging.

each cluster), but each problem has a reduced number of stop locations. Therefore, there is a trade-off between computing a few TSPMTW instances of a large size versus solving more TSPMTW instances of a reduced size.

\section{Online simulation results}

We evaluate the performance of our charging protocol in online scenarios where the energy demand evolves in time and space. The energy charging model and mobility model are the same as for offline simulations. The nodes randomly consume

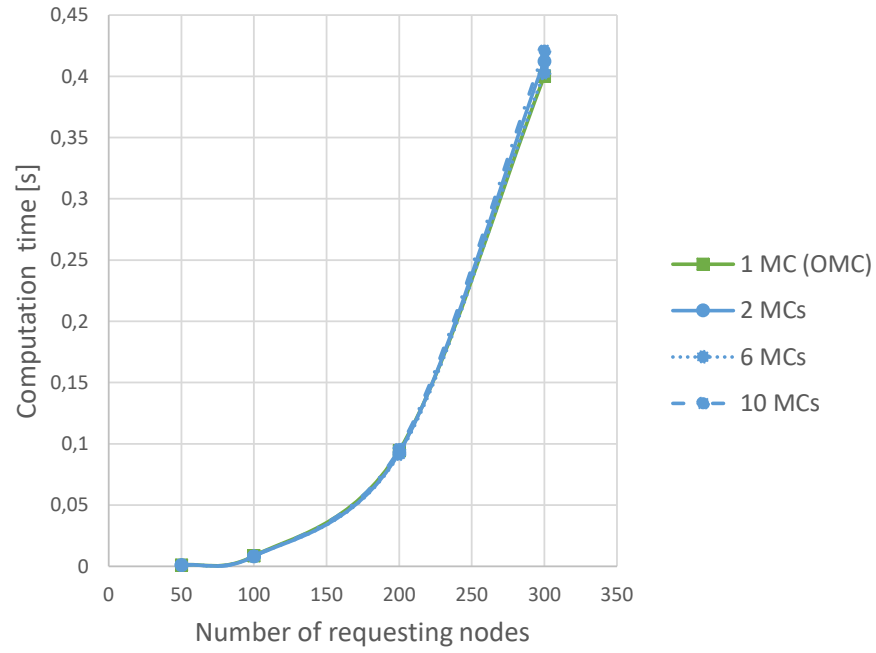

Fig. 13. Computing time of step 1 and 2.

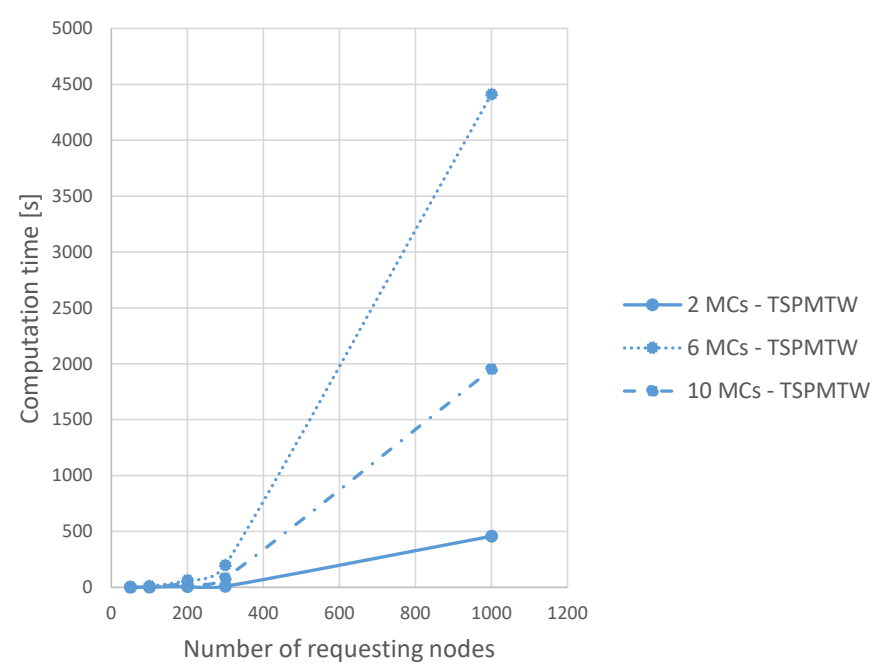

Fig. 14. Computing time of step 3 (TSPMTW).

between $10 \mathrm{~mJ}$ to $50 \mathrm{~mJ}$ per second for sensing, computation, and communication tasks in order to emulate a non-predictable node's energy consumption. The threshold values used in step 1 are $L_{c}=0.3$ and $L_{r}=0.18$.

Figure 15 illustrates the total waiting time of the chargers. First, we observe that as the number of mobile chargers increases, the waiting time also increases. This is explained by the fact that there are more chargers in the deployment area, and therefore, chargers are closer to each others, so they have to contend for charging. Second, the waiting time also increases with the number of nodes. Since there are more requesting nodes, the number of stop locations increases, and there are potentially more conflicting stop locations that cannot be visited simultaneously. Finally, as expected, the TSPMTWbased solution reduces the waiting time at stop locations compared to a TSP-based algorithm. In OMC, there is only one charger, so there is no aggregated EMR or waiting time. In CCGK, there is no waiting time, but there is significant 
aggregated EMR, as plotted in Figure 16.

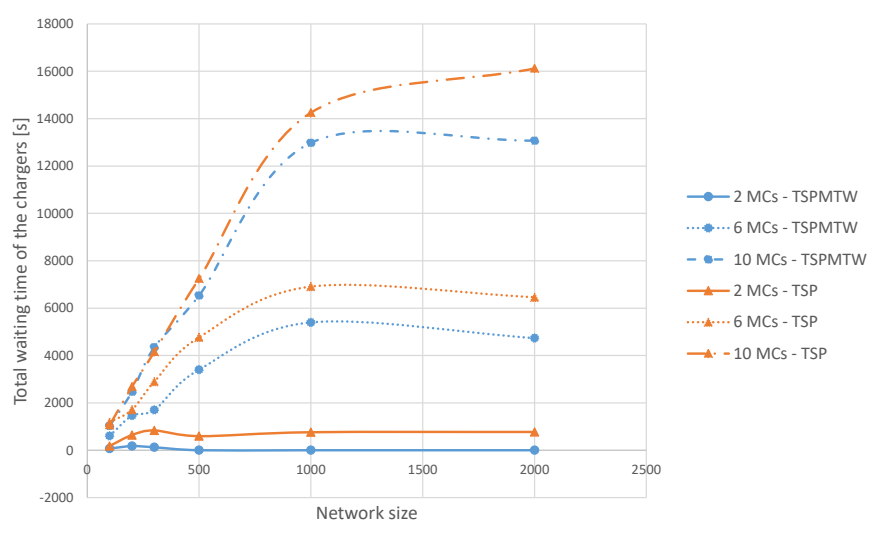

Fig. 15. Total waiting time of the chargers.

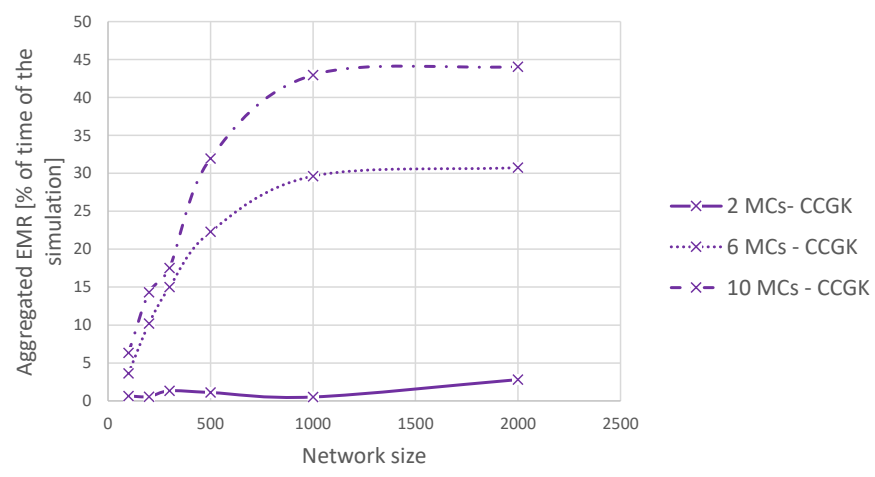

Fig. 16. Aggregated EMR.

Figure 17 shows the average failure time of network nodes. As expected, using multiple mobile chargers enables the reduction of node failures since they can charge several stop locations simultaneously if they are not conflicting. The TSPMTW-based approach improves this metric over both CCGK and the TSP. This can be explained by the fact that the TSPMTW reduces the charging delay. Indeed, the charging routes duration are minimized, and chargers can serve more demanding nodes. This is corroborated by Figure 19, which represents the number of charging tours launched during the simulation time. For identical parameters, the TSPMTW is able to complete more charging tours than CCGK, thus satisfying the energy demand of more sensors.

The energy consumed for charging by each charger is plotted in Figure 18. It is mainly influenced by the number of stop locations and the number of MCs. The more chargers there are available, the less energy a charger consumes for charging because the charging task is distributed among chargers. In contrast, as the network size increases, the charging load smoothly increases, which is in agreement with the results obtained for offline scenarios.

To better understand the evolution of the travel energy as a function of the parameter settings, it is necessary to take a look at the number of charging tours performed during a

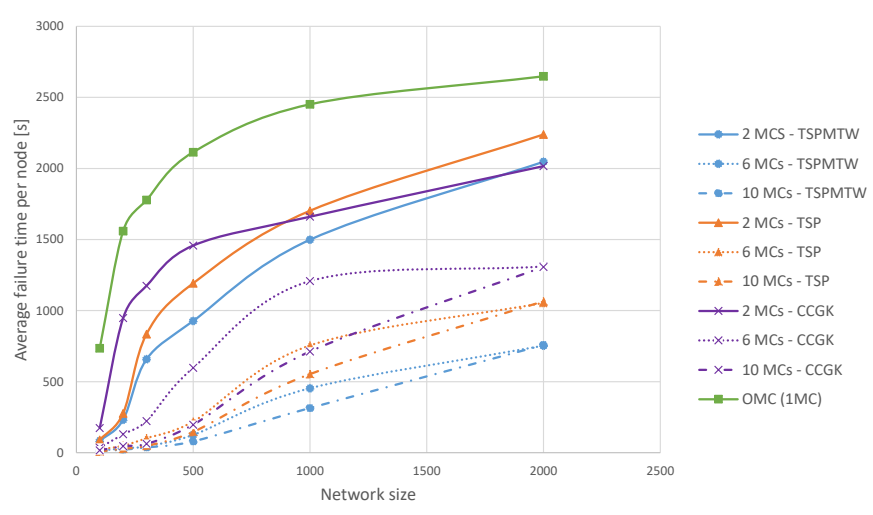

Fig. 17. Average failure time per node.

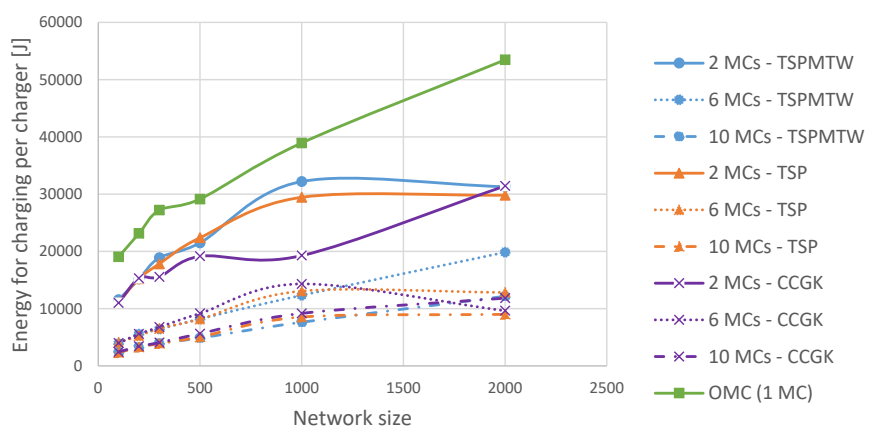

Fig. 18. Energy spent by chargers for charging.

simulation run-time. As seen in Figure 19, when the network size increases, the protocol is launched fewer times. This is because a charger's route will include more stop locations, thus lasting longer. Similarly, if there are fewer chargers in the network, each charger will have to charge more nodes and will have a longer route. A new charging tour cannot start if the previous one has not ended. These observations explain why the travel energy is directly influenced by the number of charging tours. Figure 20 shows that for a given number of charging tours, increasing the number of chargers will reduce the energy consumed per charger since demanding nodes are clustered in more groups of limited size. Finally, similar to the offline results, the TSPMTW-based solution induces a slightly increased consumption of energy for traveling compared to the TSP-based solution. We believe that it can be tolerated as long as the energy for traveling is less important than the energy needed for charging.

\section{CONCLUSION}

In this paper, we proposed a new charging protocol for wireless rechargeable sensor networks. Our approach has the specificity to simultaneously consider on-demand charging, point-to-multipoint energy transfer, multiple chargers, and electromagnetic radiation safety constraints. Our technique is based on clustering charging demands to fairly balance the charging load between chargers while minimizing the traveled distance. Then, we plan a charging path for each charger, 


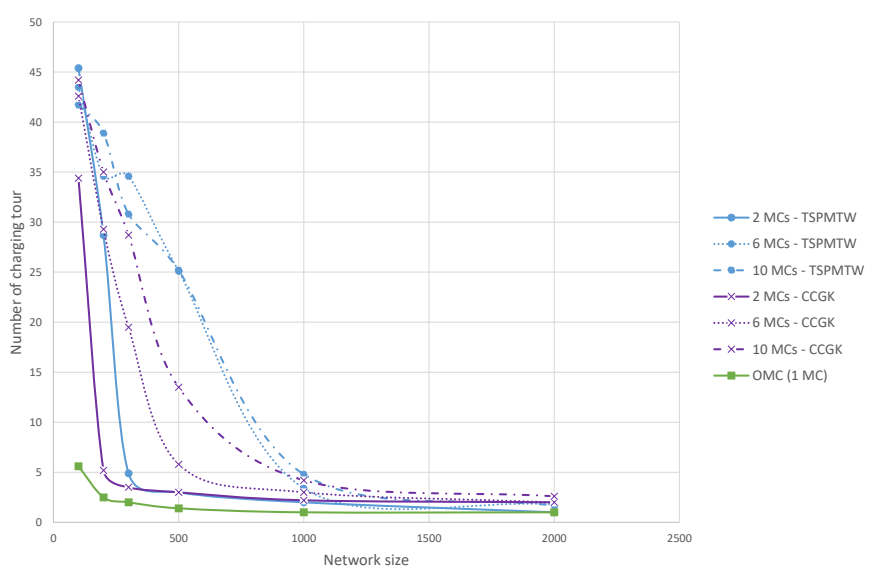

Fig. 19. Number of charging tours launched during the simulation.

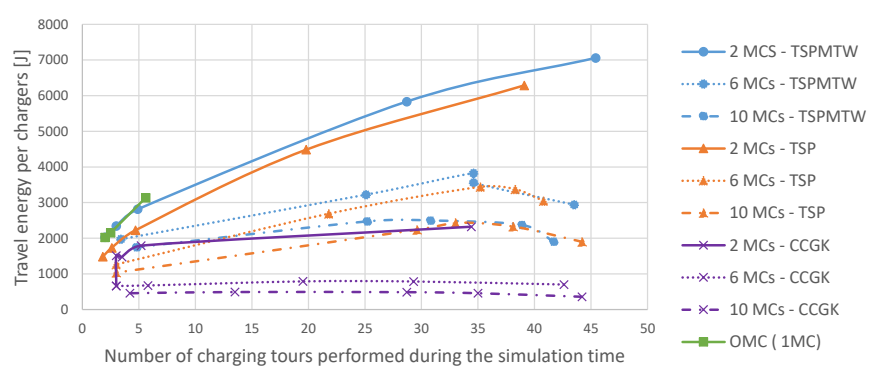

Fig. 20. Energy spent for travel in function of the number of charging tours.

ensuring that there is no aggregated EMR. If two chargers in close proximity are transferring energy simultaneously, points will exist in the deployment area that receive power from multiple chargers. Such a situation leads to aggregated EMR, which we want to prevent for health considerations. To address this particular constraint, we introduce the notion of conflicting locations. Two locations are conflicting if the power charging disks of two chargers stopping at these locations intersect. For each charger, we solve a Traveling Salesman Problem with Multiple Time Windows problem (TSPMTW). The TSPMTW problem allows us to specify the time windows during which locations can be visited, and we prevent conflicting locations from being visited at the same time.

We evaluated our scheme through extensive simulations for both online and offline scenarios. The simulation results showed that our solution can adapt to the network dynamics and achieve better performances in terms of the charging delay and node failures compared to state-of-the-art charging protocols.

\section{REFERENCES}

[1] X. Lu, P. Wang, D. Niyato, D. I. Kim, and Z. Han, "Wireless charging technologies: Fundamentals, standards, and network applications," IEEE Communications Surveys Tutorials, vol. 18, no. 2, pp. 1413-1452, Secondquarter 2016.

[2] A. Kurs, R. Moffatt, and M. Soljacic, "Simultaneous mid-range power transfer to multiple devices," Applied Physics Letters, vol. 96, 2010.
[3] C. Lin, Y. Wu, Z. Liu, M. S. Obaidat, C. W. Yu, and G. Wu, "Gtcharge: A game theoretical collaborative charging scheme for wireless rechargeable sensor networks," Journal of Systems and Software, vol. 121, pp. 88 - 104, 2016. [Online]. Available: http://www.sciencedirect.com/science/article/pii/S0164121216301480

[4] L. Xie, Y. Shi, Y. T. Hou, and H. D. Sherali, "Making sensor networks immortal: An energy-renewal approach with wireless power transfer," IEEE/ACM Transactions on Networking, vol. 20, no. 6, pp. 1748-1761, Dec 2012

[5] L. Khelladi, D. Djenouri, M. Rossi, and N. Badache, "Efficient on-demand multi-node charging techniques for wireless sensor networks," Computer Communications, vol. 101, pp. 44 - 56, 2017. [Online]. Available: http://www.sciencedirect.com/science/article/pii/S0140366416304182

[6] A. Madhja, S. Nikoletseas, and T. P. Raptis, "Distributed wireless power transfer in sensor networks with multiple mobile chargers," Computer Networks, vol. 80, pp. 89 - 108, 2015. [Online]. Available: http://www.sciencedirect.com/science/article/pii/S1389128615000298

[7] S. Nikoletseas, T. P. Raptis, and C. Raptopoulos, "Radiationconstrained algorithms for wireless energy transfer in ad hoc networks," Computer Networks, vol. 124, pp. 1 - 10, 2017. [Online]. Available: http://www.sciencedirect.com/science/article/pii/S1389128617302323

[8] H. Dai, Y. Liu, G. Chen, X. Wu, and T. He, "Safe charging for wireless power transfer," in IEEE International Conference on Computer Communications (INFOCOM), April 2014, pp. 1105-1113.

[9] L. Xie, Y. Shi, Y. T. Hou, W. Lou, H. D. Sherali, and S. F. Midkiff, "On renewable sensor networks with wireless energy transfer: The multinode case," in 9th Annual IEEE Communications Society Conference on Sensor, Mesh and Ad Hoc Communications and Networks (SECON), June 2012, pp. 10-18.

[10] _ _ "Bundling mobile base station and wireless energy transfer: Modeling and optimization," in IEEE International Conference on Computer Communications (INFOCOM), April 2013, pp. 1636-1644.

[11] Y. Shu, H. Yousefi, P. Cheng, J. Chen, Y. J. Gu, T. He, and K. G. Shin, "Near-optimal velocity control for mobile charging in wireless rechargeable sensor networks," IEEE Transactions on Mobile Computing, vol. 15 , no. 7, pp. 1699-1713, July 2016.

[12] C. Lin, G. Wu, M. S. Obaidat, and C. W. Yu, "Clustering and splitting charging algorithms for large scaled wireless rechargeable sensor networks," Journal of Systems and Software, vol. 113, pp. 381 - 394, 2016.

[13] X. Ren, W. Liang, and W. Xu, "Maximizing charging throughput in rechargeable sensor networks," in 23rd International Conference on Computer Communication and Networks (ICCCN), Aug 2014, pp. 18.

[14] C. M. Angelopoulos, S. Nikoletseas, and T. P. Raptis, "Wireless energy transfer in sensor networks with adaptive, limited knowledge protocols," Computer Networks, vol. 70, pp. 113 - 141, 2014. [Online]. Available: http://www.sciencedirect.com/science/article/pii/S1389128614001972

[15] L. He, L. Fu, L. Zheng, Y. Gu, P. Cheng, J. Chen, and J. Pan, "Esync: An energy synchronized charging protocol for rechargeable wireless sensor networks," in Proceedings of the 15th ACM International Symposium on Mobile Ad Hoc Networking and Computing, ser. MobiHoc '14. New York, NY, USA: ACM, 2014, pp. 247-256. [Online]. Available: http://doi.acm.org/10.1145/2632951.2632970

[16] W. Xu, W. Liang, X. Jia, and Z. Xu, "Maximizing sensor lifetime in a rechargeable sensor network via partial energy charging on sensors," in 13th Annual IEEE International Conference on Sensing, Communication, and Networking (SECON), 06 2016, pp. 1-9.

[17] P. Zhong, Y. Zhang, S. Ma, X. Kui, , and J. Gao, "Rcss: A real-time on-demand charging scheduling scheme for wireless rechargeable sensor networks," Sensors, vol. 18, 2018.

[18] C. Lin, Y. Zhou, H. Dai, J. Deng, and G. Wu, "Mpf: Prolonging network lifetime of wireless rechargeable sensor networks by mixing partial charge and full charge," in 15th Annual IEEE International Conference on Sensing, Communication, and Networking (SECON), June 2018, pp. $1-9$.

[19] C. Wang, J. Li, F. Ye, and Y. Yang, "Multi-vehicle coordination for wireless energy replenishment in sensor networks," in IEEE 27th International Symposium on Parallel and Distributed Processing, May 2013, pp. 1101-1111.

[20] L. Jiang, X. Wu, G. Chen, and Y. Li, "Effective on-demand mobile charger scheduling for maximizing coverage in wireless rechargeable 
sensor networks," Mobile Networks and Applications, vol. 19, pp. 543551,082014

[21] G. Jiang, S.-K. Lam, Y. Sun, L. Tu, and J. Wu, "Joint charging tour planning and depot positioning for wireless sensor networks using mobile chargers," IEEE/ACM Transactions on Networking, vol. 25, pp. 2250-2266, 2017.

[22] H. Dai, Y. Liu, G. Chen, X. Wu, and T. He, "Scape: Safe charging with adjustable power," in IEEE 34th International Conference on Distributed Computing Systems, June 2014, pp. 439-448.

[23] H. Dai, Y. Liu, A. X. Liu, L. Kong, G. Chen, and T. He, "Radiation constrained wireless charger placement," in IEEE International Conference on Computer Communications (INFOCOM), April 2016, pp. 1-9.

[24] J. Hurkala, "Time-dependent traveling salesman problem with multiple time windows," in FedCSIS Position Papers, 2015.

[25] C. de Jong, G. Kant, and A. van Vliet, "On Finding Minimal Route Duration in the Vehicle Routing Problem with Multiple Time Windows," Econometric Institute, Erasmus University Rotterdam, Rotterdam, project ALCOM IT, 1998.

[26] G. Pesant, M. Gendreau, J.-Y. Potvin, and J.-M. Rousseau, "On the flexibility of constraint programming models: From single to multiple time windows for the traveling salesman problem," European Journal of Operational Research, vol. 117, no. 2, pp. 253 - 263, 1999. [Online]. Available: http://www.sciencedirect.com/science/article/pii/S0377221798002483

[27] "Google optimization tools," accessed: 2017-02-14. [Online]. Available: https://developers.google.com/optimization 